\title{
High-quality Dicing of Semiconductor Package by Superposition of Pulsed Fiber Laser and SHG:YAG Laser
}

\author{
Hibiki YAMAMOTO ${ }^{1}$, Yasuhiro OKAMOTO ${ }^{2}$, Muhamad Fahmi bin Mohd Noor ${ }^{1}$, \\ Ryoji KITADA $^{1}$ and Akira OKADA ${ }^{3}$ \\ ${ }^{1}$ Okayama University, 3-1-1 Tsushimanaka, Kita-ku, Okayama 700-8530, Japan, \\ hyamamoto@ntmlab.mech.okayama-u.ac.jp \\ ${ }^{2}$ Okayama University, okamoto@mech.okayama-u.ac.jp \\ ${ }^{3}$ Okayama University, okada@mech.okayama-u.ac.jp
}

\begin{abstract}
:
The application of laser beam processing has been expected in the field of semiconductor devices because of its flexible cutting line and narrow kerf width. However, the deterioration of cutting quality would occur due to the thermal damage, since the laser beam processing is mainly thermal process. Therefore, the laser dicing method by the superposition of fiber laser and SHG:YAG laser was proposed to perform the high-quality processing. The smaller kerf width could be achieved by controlling the time delay between both laser pulses, and the synchronized laser pulses led to the smaller heat affected zone.
\end{abstract}

Keywords: Laser dicing, Semiconductor package, Superposition, Fiber laser, SHG:YAG laser

\section{Introduction}

Recently, the manufacturing technology of semiconductor devices has been remarkably developed with demands for miniaturization and lightning of many products. The molding method of silicon chip has been changed from the discrete package molding method to the solid type molding one, in which many chips are molded collectively. Solid type molded semiconductor package is required to separate into an individual package after molding. However, the current blade dicing method for the separation is only available for the straight cutting, and it is difficult to cut the required shape of current electronics device with complicated lines. Moreover, it is difficult to apply the dicing method to the small size package. Because, a kerf width is relatively large due to the thickness of blade, and it is hard to mount the small separated package.

On the other hand, the laser beam processing has been expected as a new dicing method, since it has high potential such as flexible cutting line and narrow kerf width. However, the deterioration of cutting quality due to thermal damage is still remained as a problem, since the laser beam processing is mainly carried out by the thermal removal, in which the temperature of material rises due to the absorption of laser beam. Previous works made it clear that the thermal damage was decreased by supplying the cooled gas to the processing point ${ }^{1)}$. However, the processing head became the complex configuration to prevent the frost from adhering to the optics. Therefore, the high quality laser dicing method for the semiconductor package is required with a small heat affected zone without the cooled assist gas.

In this study, the laser dicing method for the semiconductor package by the superposition of fiber laser and SHG:YAG laser was proposed. The fiber laser can be focused in a small spot because of its excellent beam quality, and SHG:YAG laser has the potential to perform a laser dicing with a small heat affected zone because of its high photon energy ${ }^{2)}$. Figure 1 schematically shows the expected effect of superposition, in which the substrate of the workpiece will be removed by SHG:YAG laser and the fiber laser will cut throughout the epoxy-resin of the workpiece. It would be expected by the different wavelength and thermal properties between the substrate and the epoxy-resin of the workpiece. Therefore, the high quality laser dicing could be expected by the superposition of a fiber laser and a SHG:YAG laser. Since the both laser beams are a pulsed laser, the synchronization or a time delay between both laser pulses would have an effect on cutting characteristics. Hence, the laser dicing by the superposition of two laser beams with the various time delays between both laser pulses was been experimentally investigated, and its cutting characteristics were discussed in this study.

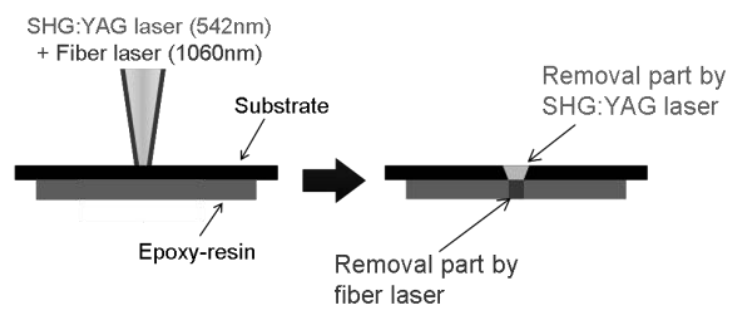

Figure 1: Effect of superposition.

\section{Experimental setup}

\subsection{Time delay management and cutting method}

Figure 2 shows a schematic illustration of experimental setup for a time delay management in the superposition of fiber laser and SHG:YAG laser. The time delay between both laser pulses was controlled by a digital delay generator (SRS DG645). Two photodiodes were used in order to detect a transmitted light. A first photodiode was set behind the reflective mirror in order to detect the fiber laser, and its signal was used as the external trigger for 


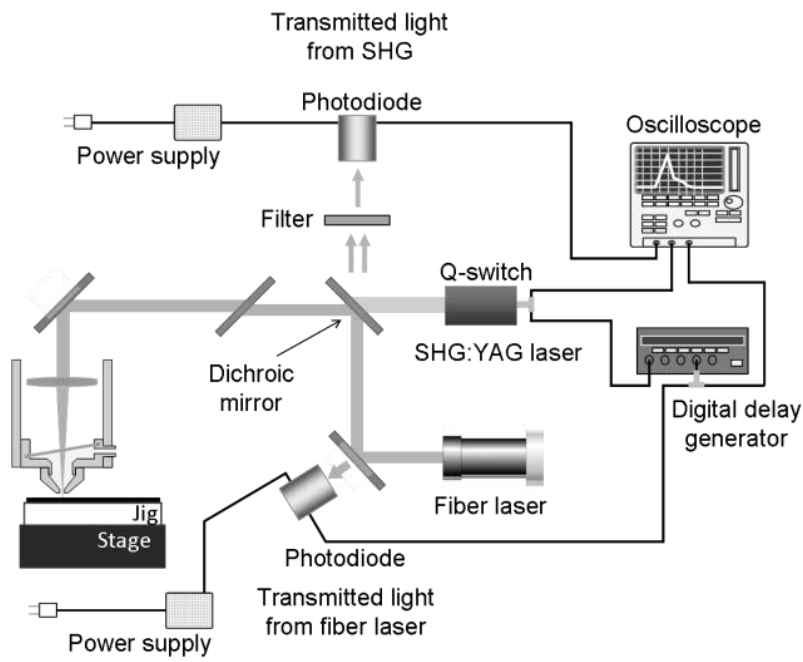

Figure 2: Schematic diagram of experimental setup for time delay management.

Table 1: Cutting conditions.

\begin{tabular}{|c|c|c|c|c|}
\hline $\begin{array}{l}\text { Pulse repetition rate of } \\
\text { Fiber laser }\end{array}$ & $20 \mathrm{kHz}$ & $20 \mathrm{kHz}$ & $20 \mathrm{kHz}$ & $20 \mathrm{kHz}$ \\
\hline $\begin{array}{l}\text { Pulse repetition rate of } \\
\text { SHG:YAG laser }\end{array}$ & - & $20 \mathrm{kHz}$ & $10 \mathrm{kHz}$ & $10 \mathrm{kHz}$ \\
\hline $\begin{array}{l}\text { Average power of } \\
\text { fiber laser }\end{array}$ & $16 \mathrm{~W}$ & $16 \mathrm{~W}$ & $16 \mathrm{~W}$ & $16 \mathrm{~W}$ \\
\hline $\begin{array}{l}\text { Average power of } \\
\text { SHG YAG laser }\end{array}$ & - & $8 \mathrm{~W}$ & $8 \mathrm{~W}$ & $4 \mathrm{~W}$ \\
\hline Feed rate, $\mathrm{F}$ & \multicolumn{4}{|c|}{$5,10,15,20 \mathrm{~mm} / \mathrm{s}$} \\
\hline Time delay, $T \mathrm{~d}$ & - & $\begin{array}{c}0,1,2,5,10,20 \\
25,30,40 \mu \mathrm{s}\end{array}$ & $0,25 \mu \mathrm{s}$ & $0,25 \mu \mathrm{s}$ \\
\hline
\end{tabular}

digital delay generator. The second photodiode was set behind the dichroic mirror in order to detect a transmitted SHG:YAG laser. Since the fiber laser and SHG:YAG laser were superposed at the dichroic mirror, an additional filter was set at the front of the second photodiode in order to detect the only SHG:YAG laser.

Table 1 shows the experimental conditions, and Figure 3 shows the definition of time delay $T \mathrm{~d}$ between both laser pulses. In the experiment with fiber laser $16 \mathrm{~W}$ at $20 \mathrm{kHz}$ and SHG:YAG laser $8 \mathrm{~W}$ at $20 \mathrm{kHz}$ or $10 \mathrm{kHz}$, the temporal positions of both laser pulses were adjusted at the same temporal position $(T \mathrm{~d}=0 \mu \mathrm{s})$ to investigate the effect of different pulse repetition rate under the same average power. Moreover, SHG:YAG laser $4 \mathrm{~W}$ at $10 \mathrm{kHz}$ and $8 \mathrm{~W}$ at $20 \mathrm{kHz}$ were used to discuss the cutting results under the same pulse energy condition. Under the same laser irradiation conditions mentioned above, the time delay $T \mathrm{~d}=25 \mu \mathrm{s}$, which means that pulses of SHG:YAG laser locate at the middle of pulses of fiber laser, was also used. Furthermore, experiments were carried out in order to investigate of various time delays between both laser pulses with SHG:YAG laser $8 \mathrm{~W}$ at $20 \mathrm{kHz}$. Nitrogen was used as an assist gas at $600 \mathrm{kPa}$ from the straight throat nozzle of $1.0 \mathrm{~mm}$ diameter.

\subsection{Semiconductor package}

The semiconductor package was used as a workpiece

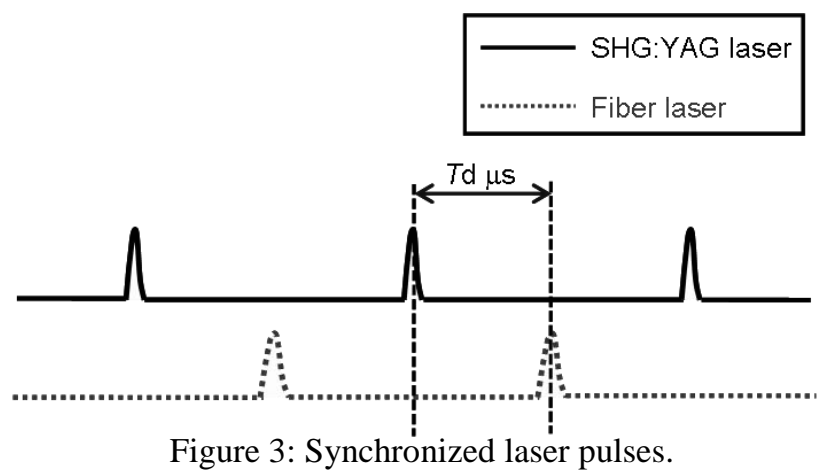

Table 2: Properties of substrate and epoxy resin.

\begin{tabular}{l|l|l}
\hline Material & Substrate & Epoxy resin \\
\hline Pyrolysis temperature $\left[{ }^{\circ} \mathrm{C}\right]$ & 350 & 350 \\
\hline Thermal conductivity $[\mathrm{W} /(\mathrm{m} \cdot \mathrm{K})]$ & 0.41 & 0.7 \\
\hline Density $\left[\mathrm{g} / \mathrm{cm}^{3}\right]$ & 1.8 & 1.84 \\
\hline Specific heat $[\mathrm{J} /(\mathrm{kg} \cdot \mathrm{K})]$ & 800 & $799-1146$ \\
\hline
\end{tabular}

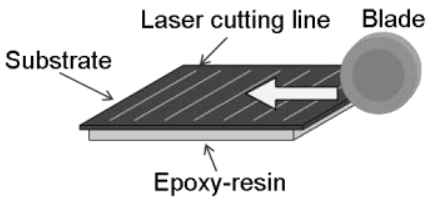

(a) Blade dicing.

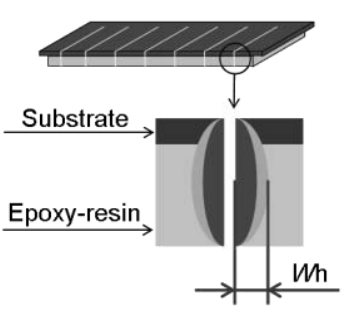

(b) Measurement of heat HAZ after dicing.
Figure 4: Evaluation method for heat affected zone on laser cut surface.

which is consisted of thermosetting epoxy-resin with silica for molding compounds and glass epoxy board for semiconductor package with insulator coatings $\mathrm{s}^{3), 4)}$. The thickness of substrate and epoxy-resin are $0.25 \mathrm{~mm}$ and $0.80 \mathrm{~mm}$, respectively. Main characteristics of the substrate and the epoxy-resin are shown in Table 2.

\subsection{Evaluation method of cross section}

In order to evaluate the cross section, the blade dicing was used to cut through the workpiece as shown in Figure 4. The observation and measurement were mainly carried out by using an optical microscope (Keyence VH-7000). The width of discolored area on the cut surface was measured, and the average of 14 points measured data was defined as the width of heat affected zone (HAZ) Wh. Both brown and black colors were observed in the HAZ.

\section{Experimental results and discussion \\ 3.1 Evaluation of kerf line}

The micrographs of cut kerf with only fiber laser $16 \mathrm{~W}$ at $20 \mathrm{kHz}$, synchronized, $25 \mu$ s delayed laser pulses at $8 \mathrm{~W}$ and $20 \mathrm{kHz}$ SHG:YAG laser were shown in Figure 5. As can be seen from the figure, the deterioration of cut edge quality of substrate side was observed with the increment of feed rate in the case of fiber laser. On the other hand, in both synchronized and $25 \mu \mathrm{s}$ delayed laser pulses, the straight kerf line was obtained even at the high feed rate. 


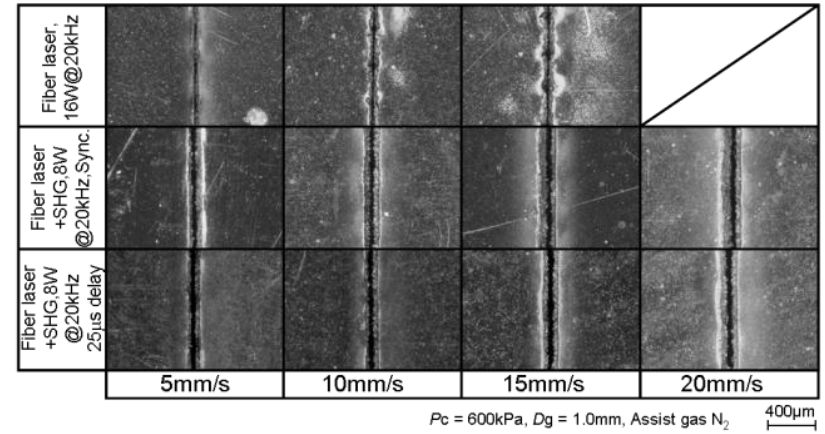

Figure 5: Micrographs of kerf for various feed rates.

Table 3: Cut edge quality.

$\bigcirc:$ Straight kerf line, $\triangle$ : Deterioration of cut edge

$X$ : Not be accomplished a full cut of workpiece

\begin{tabular}{|c|c|c|c|c|c|}
\hline \multicolumn{2}{|c|}{ Laser condition $\quad$ Feed rate } & $5 \mathrm{~mm} / \mathrm{s}$ & $10 \mathrm{~mm} / \mathrm{s}$ & $15 \mathrm{~mm} / \mathrm{s}$ & $20 \mathrm{~mm} / \mathrm{s}$ \\
\hline \multicolumn{2}{|c|}{ Fiber laser, 16W@20kHz } & $\bigcirc$ & $\triangle$ & $\triangle$ & $\times$ \\
\hline \multirow{2}{*}{$\begin{array}{c}\text { Fiber laser, 16W } \\
\text { @ 20kHz + } \\
\text { SHG, 8W@20kHz }\end{array}$} & Sync. & 0 & 0 & $\bigcirc$ & 0 \\
\hline & $25 \mu$ s delay & $\bigcirc$ & $\bigcirc$ & 0 & 0 \\
\hline \multirow{2}{*}{$\begin{array}{c}\text { Fiber laser, 16W } \\
@ 20 \mathrm{kHz}+ \\
\text { SHG, 8W@10kHz }\end{array}$} & Sync. & $\bigcirc$ & $\bigcirc$ & $\bigcirc$ & $\bigcirc$ \\
\hline & $25 \mu$ s delay & $\bigcirc$ & $\bigcirc$ & $\bigcirc$ & $\bigcirc$ \\
\hline \multirow{2}{*}{$\begin{array}{c}\text { Fiber laser, 16W } \\
\text { @ } 20 \mathrm{kHz}+ \\
\text { SHG, 4W@10kHz }\end{array}$} & Sync. & $\bigcirc$ & O & $\bigcirc$ & $\times$ \\
\hline & $25 \mu$ s delay & $\bigcirc$ & $\bigcirc$ & $\bigcirc$ & $\times$ \\
\hline
\end{tabular}

Table 3 shows results of cut edge quality whether the kerf line is straight under various processing conditions. As shown in the table, the superposition of fiber laser and SHG:YAG laser $4 \mathrm{~W}$ at $10 \mathrm{kHz}$ could not accomplish a full cut of the workpiece at the feed rate $20 \mathrm{~mm} / \mathrm{s}$, while the full cut could be performed by the combination of fiber laser and SHG:YAG laser 8W. On the other hand, every synchronized and $25 \mu \mathrm{s}$ delayed laser pulses conditions could perform the straight kerf line. It is considered that the substrate side would be removed by SHG:YAG laser, and the fiber laser would cut throughout the epoxy-resin side. In addition, the result showed that the pulse repetition rate and the average power of SHG:YAG laser were important factors and useful parameters to obtain a maximum cutting speed.

Figure 6 shows the kerf width of the substrate side for various feed rates by the synchronized or $25 \mu$ s delayed laser pulses under the different repetition rate and the same average power condition. In both synchronized and $25 \mu$ s time delay laser pulse, the kerf width of the substrate side increased with increasing the feed rate, since the absorbed energy into the substrate side per a pulse increased with decreasing the overlap rate. The kerf width of the substrate side was larger by the superposition of SHG:YAG laser at $10 \mathrm{kHz}$, compared with SHG:YAG laser at $20 \mathrm{kHz}$, under the $8 \mathrm{~W}$ average power condition. It is considered that the different of pulse energy of SHG:YAG laser and overlap rate would lead to the differences of kerf width at the substrate side.

In order to investigate the cutting characteristics under the same pulse energy condition of SHG:YAG laser, the superposition of SHG:YAG laser $4 \mathrm{~W}$ at $10 \mathrm{kHz}$ and $8 \mathrm{~W}$ at

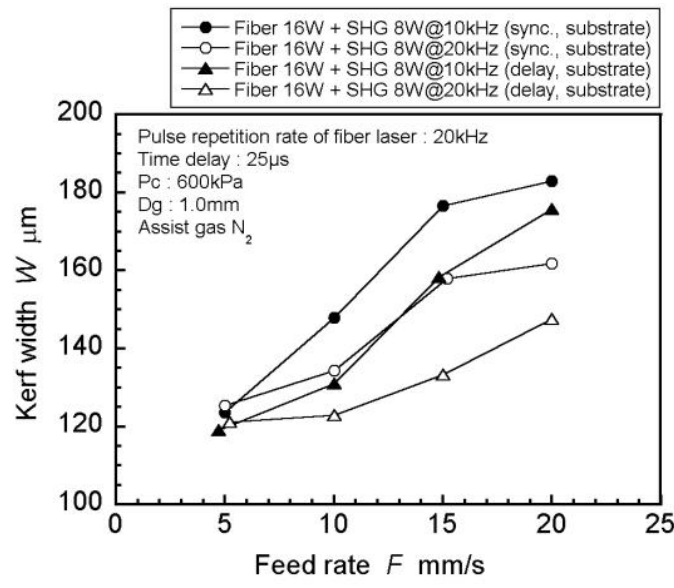

Figure 6: Kerf width for various feed rates by synchronized and $25 \mu$ s time delays under the same average power.

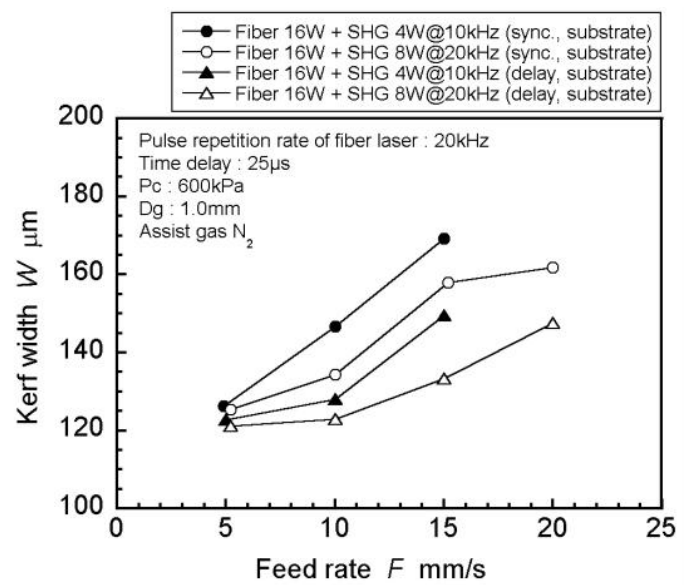

Figure 7: Kerf width for various feed rates by synchronized and $25 \mu$ s time delays under the same pulse energy.

$20 \mathrm{kHz}$ were carried out by synchronized or $25 \mu$ s time delay irradiation, and the results are shown in Figure 7. As can be seen from the figure, the kerf width of the substrate side by superposition SHG:YAG laser $4 \mathrm{~W}$ at $10 \mathrm{kHz}$ is still larger than that with SHG:YAG laser $8 \mathrm{~W}$ at $20 \mathrm{kHz}$ in both synchronized and $25 \mu$ s time delay between both laser pulses. The peak power of SHG:YAG laser might be changed by the pulse repetition rate, since the pulse width of SHG:YAG laser at $10 \mathrm{kHz}$ is smaller than that at $20 \mathrm{kHz}$. Since the peak power will be lower at the long pulse width under the same pulse energy, the power intensity will be decreased. Therefore, the lower peak power led to a smaller kerf width because of low power intensity.

As can be seen from Figures 6 and 7, the kerf width by $25 \mu$ s time delay between both laser pulses is smaller than that by synchronized laser pulses. It is assumed that the diffusion of absorbed energy on the surface side would be higher in the case of synchronized laser pulses, in which both laser beams were irradiated at the same time.

The variations of the kerf width with time delay are shown in Figure 8. As shown in the figure, the kerf width does not change greatly by the time delay. However, the kerf width by time delayed laser pulse is smaller than that by synchronized laser pulse $(T \mathrm{~d}=0)$. 


\subsection{Evaluation of cross section}

The comparison of heat affected zones by synchronized laser pulses and $25 \mu$ s time delay between both laser pulses is shown in Figure 9. As can be seen from the figure, due to the high photon energy, the superposition of fiber laser and SHG:YAG laser might affect the reduction of heat affected zone, compared with that only by the fiber laser. On the other hand, the reduction rate of heat affected zone by the superposition of SHG:YAG laser $8 \mathrm{~W}$ at $20 \mathrm{kHz}$ is smaller than SHG:YAG laser $8 \mathrm{~W}$ at $10 \mathrm{kHz}$ in the case of synchronized laser pulses and $25 \mu$ s time delay between both laser pulses. The same result was also obtained under the same pulse energy condition of SHG:YAG laser by superposition of the fiber laser with SHG:YAG laser $4 \mathrm{~W}$ at $10 \mathrm{kHz}$. It is considered that overlap rate and pulse energy might have a great influence on the size of heat affected zone. The almost energy of SHG:YAG laser had been used to remove the substrate side in the case of low overlap rate or pulse energy. Therefore, the absorbed energy on the epoxy-resin side became smaller, and the heat affected zone would decrease.

On the other hand, it can be seen that synchronized laser pulses could lead to the reduction of heat affected zone under the every SHG:YAG laser condition. In the case of synchronized laser pulses, since the energy of fiber laser and SHG:YAG laser had been used to remove the material of top surface, the remaining energy was smaller. Therefore, the heat affected zone would decrease.

Figure 10 shows the size of heat affected zone for various time delays between both laser pulses. As shown in figure, the width of heat affected zone was equivalent under every time delay condition. However, the width of heat affected zone by synchronized laser pulses is smaller than that by various time delays between both laser pulses. Therefore, the synchronized laser pulses would lead to a smaller heat affected zone compared with that by time delayed laser pulses.

\section{Conclusions}

The high quality laser dicing for the semiconductor package by the superposition of fiber laser and SHG:YAG laser was proposed, and its cutting characteristics were experimentally investigated.

Main conclusions obtained in this study are as follows:

(1) The laser dicing by the superposition of fiber laser and SHG:YAG laser with the high pulse repetition rate led to a straighter kerf with smaller width under the same pulse energy condition.

(2) The smaller kerf width could be achieved by controlling the time delay between both laser pulses compared with synchronized laser pulses.

(3) The laser dicing by the superposition of fiber laser and SHG:YAG laser could reduce the heat affected zone. Moreover, the synchronized laser pulses could lead to the smaller heat affected zone, compared with the time delayed laser pulses.

\section{Acknowledgement}

The authors are grateful to TOWA Corporation for supplying semiconductor packages.

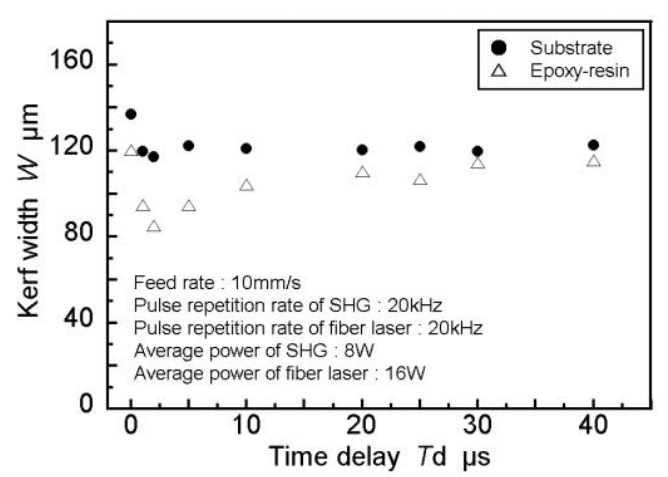

Figure 8: Kerf width for various time delays between both laser pulses.

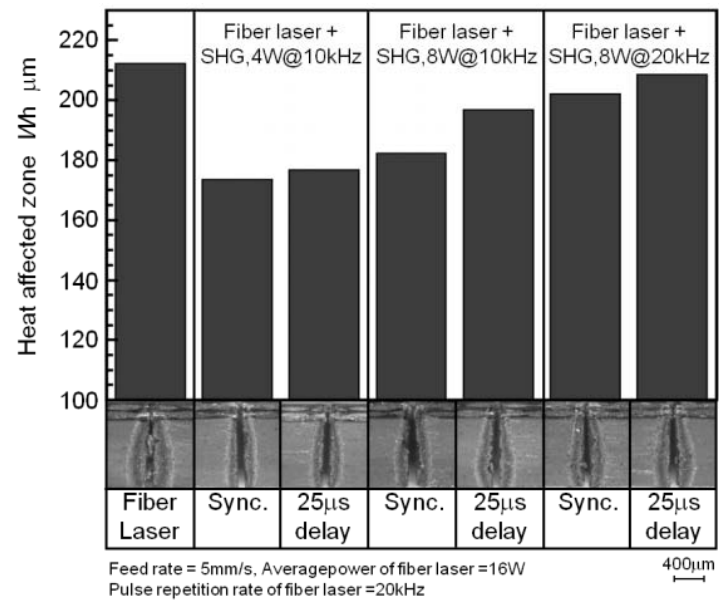

Figure 9: Micrographs of cross section and heat affected zone size.

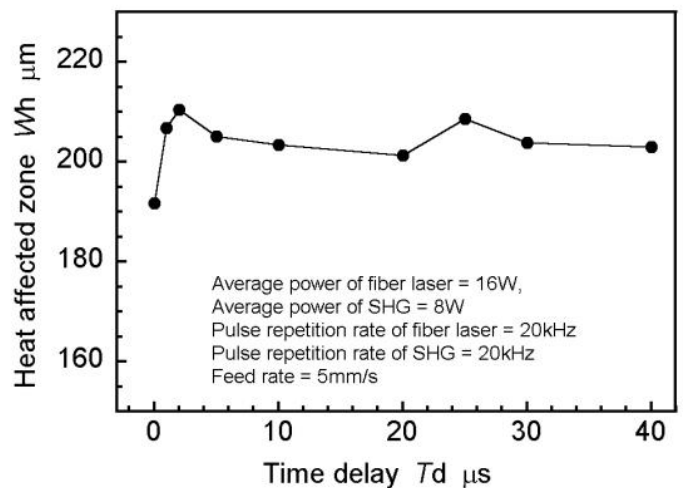

Figure 10: Heat affected zone size for various time delays between both laser pulses.

\section{References}

[1] Kitada, R., Okamoto, Y., Uno, Y., Ikeda, T., 2010, High Quality Laser Dicing of Semiconductor Package with a Single-mode Q-switch Fiber Laser, IJEM, No. 15, pp.33-38.

[2] Miyamoto, I., Seo-Jeong, P., Kosumi, T., Watanabe, K., Ooie, T., 2004, Applications of Single-Mode Fiber-Lasers to Microfabrication, Proc. of the 61th Conference JLPS, pp30-40 (in Japanese).

[3] Kakimoto, M., Takahashi, A., 2010, The Development of a Substrate Material for Electronics Packaging, pp94-109 (in Japanese).

[4] The Japan Society of Epoxy-Resin Technology, 2003, General Remarks of Epoxy-Resin, Vol. 3, App. 1, pp133-150 (in Japanese). 